\title{
Teodor: A semi-autonomous search and rescue and demining robot
}

\author{
DE CUBBER Geert ${ }^{1, a}{ }^{*}$, BALTA Haris ${ }^{1, b}$ and LIETART Claude ${ }^{1, c}$ \\ ${ }^{1}$ Royal Military Academy, Department of Mechanics, Unmanned Vehicle Centre, \\ Avenue de la Renaissance 30, 1000 Brussels, Belgium \\ a ${ }^{*}$ geert.de.cubber@rma.ac.be, ${ }^{b}$ haris.balta@rma.ac.be, ${ }^{c}$ claude.lietart@rma.ac.be
}

Keywords: Mechanical Design, Robotics, Search and Rescue, Environment Monitoring and Perception, Humanitarian Demining.

\begin{abstract}
In this paper, we present a ground robotic system which is developed to deal with rough outdoor conditions. The platform is to be used as an environmental monitoring robot for 2 main application areas: 1) Humanitarian demining: The vehicle is equipped with a specialized multichannel metal detector array. An unmanned aerial system supports it for locating suspected locations of mines, which can then be confirmed by the ground vehicle. 2) Search and rescue: The vehicle is equipped with human victim detection sensors and a $3 \mathrm{D}$ camera enabling it to assess the traversability of the terrain in front of the robot in order to be able to navigate autonomously. This paper discusses both the mechanical design of these platforms as the autonomous perception capabilities on board of these vehicles.
\end{abstract}

\section{Introduction}

In recent times, mobile robots are more and more leaving the protected lab environment and entering the unstructured and complex outside world. However, the rough outside world poses several constraints on the mechanical structure of the robotic system, on the electronics and the control architecture and on the robustness of the autonomous components.

Main factors to keep into consideration for the design of ground robotics systems are:

- Mobility on difficult terrain and different soils

- Resistance to rain and dust

- Capability of working in changing illumination conditions and in direct sunlight

- Capability of dealing with unreliable communication links, requiring autonomous navigation capabilities

- Long range autonomy

The constraints imposed by the rough outside world are often too hard for platforms stemming from scientific laboratories. Hence, more robust and proven mechanical designs need to be used. Next to the technical constraints, an important factor to be taken into consideration is also the economic opportunity of developing a mechanical design for a robotic platform which is focused on one sole task or type of environment, as there may not be a wide enough market for the valorization of this platform. Hence, often platforms with a wider envelope of capabilities and with more flexible capacities are selected, even though these platforms are not always the best for a single task.

In the domain of search and rescue robotics, a considerable amount of previous work [1] has explored the applicability of unmanned systems for assisting human search and rescue workers. An interesting initiative to note is that under the initiative of the American National Institute of Standards and Technology (NIST), standardized test and evaluation methodologies have been devised for these types of robots [2], allowing to benchmark the performance of systems next to one another.

In the domain of humanitarian demining robots, no such standardized test and evaluation methodology exists yet, even though many of the NIST-test could be ported to humanitarian demining robots. Previous work on humanitarian demining robots has concentrated for a long time on the conception of walking prototypes [3,4,5], due to the advantages of a redundant gait 
placement strategy on uncertain soil. However, due to the many difficulties confronted when working with (fragile) walking prototypes on rough outdoor terrain, more and more wheeled systems are now being explored and put to the test $[6,7,8]$.

From an operational point of view, the requirements for search and rescue and demining robots are very steep $[9,10,11]$ :

- The platform has to be able to cope with different types of terrain (mud, gravel, grass, rocks, sand ...). To ensure mobility on all these types of terrain, a tracked system was chosen.

- The platform has to be able to cope with steep hills and be able to carry a heavy sensor payload. This requirement imposes important constraints on the motor power and the power supply. Currently, the robot tracks are powered by an electromotor, allowing the robot to climb stairs and ramps up to 45 degrees.

- The platform has to be rain resistant. A covering shelter was developed, housing all the electronics and on-board processing mechanisms of the robot.

- The platform has to be able to move precisely, mainly for mine localization. Due to this constraint, the top speed of the platform is rather low $(3.0 \mathrm{~km} / \mathrm{h})$

- The platform must be capable of carrying an extensive and flexible sensory payload, depending on the application. The payload of the proposed platform is about $200 \mathrm{~kg}$ for a base platform weight including the batteries of about $350 \mathrm{~kg}$.

- The platform must be able to operate for 2 to 3 hours in "normal" operation conditions.

Based on these requirements, the Teodor platform was chosen. The Telerob Teodor [12], shown on Fig. 1, is a standard Explosive Ordnance Disposal robot. In its standard configuration, the robot is equipped with a powerful manipulator arm, but as this arm is less useful for deminingand search and rescue applications, this manipulator arm was removed here.

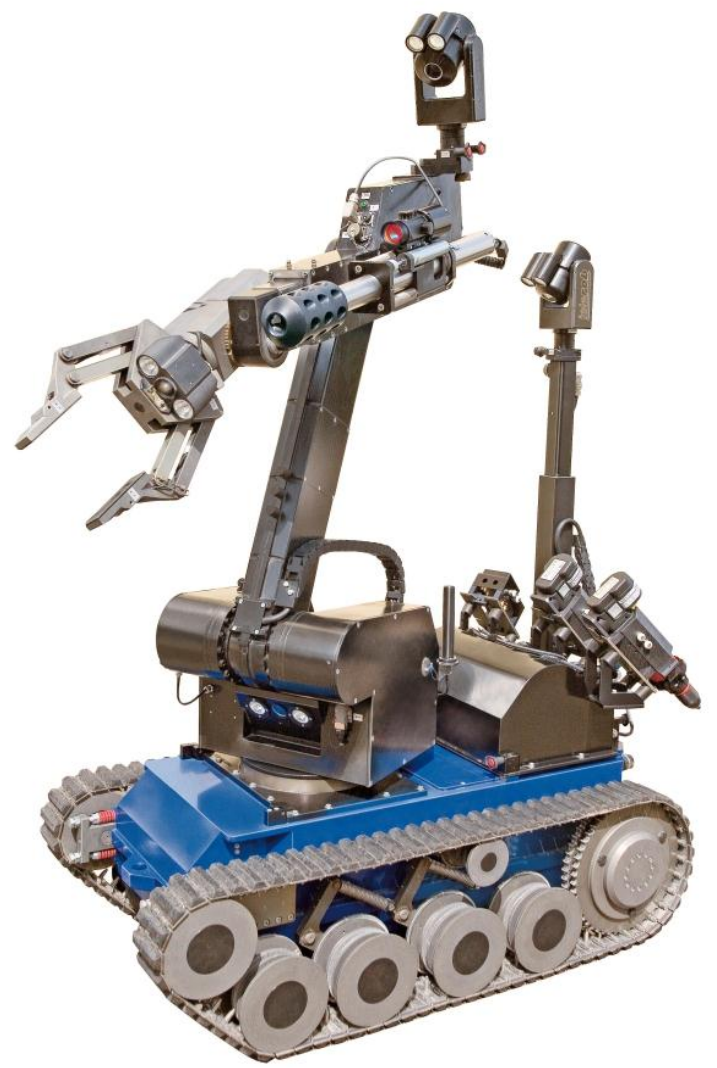

Fig. 1: Teodor Explosive Ordnance Disposal robot used as a base platform 


\section{Demining Robot Platform}

The demining robot platform described here was developed in the context of the EU-FP7TIRAMISU project. The objective of the TIRAMISU project is to provide the Mine Action community with a toolbox to assist in addressing the many issues related to Humanitarian Demining, thus promoting peace, national and regional security, conflict prevention, social and economic rehabilitation and post-conflict reconstruction.

The tools in development are divided into three main categories:

1) Demining planning tools, which will help to locate landmines and UXOs, and define contaminated areas.

2) Detection and disposal tools, which will neutralise mines and UXOs and safeguard the lives of operators.

3) Training and Mine Risk education tools.

The developed demining robot, as depicted on Fig. 2, falls into the second category of detection tools. As shown on Fig. 2, the demining ground robot platform [13] is extended with an Unmanned Aerial System [14]. The idea of this setup is to create a collaborative aerial + ground robotic demining platform, where the airborne systems scans the minefield from above (with a visual and infrared sensor), scanning for potential locations of mines, which are then to be confirmed by the ground robotic system, which is discussed here.

The ground robotic system [13] consists of a heavily adapted Teodor robot system with as most notable feature the array of metal detectors mounted in front of the vehicle. This array of metal detectors consists of 5 detection coils, allowing to detect smaller anti-personnel mines as well as larger anti-tank mines with the same system setup.
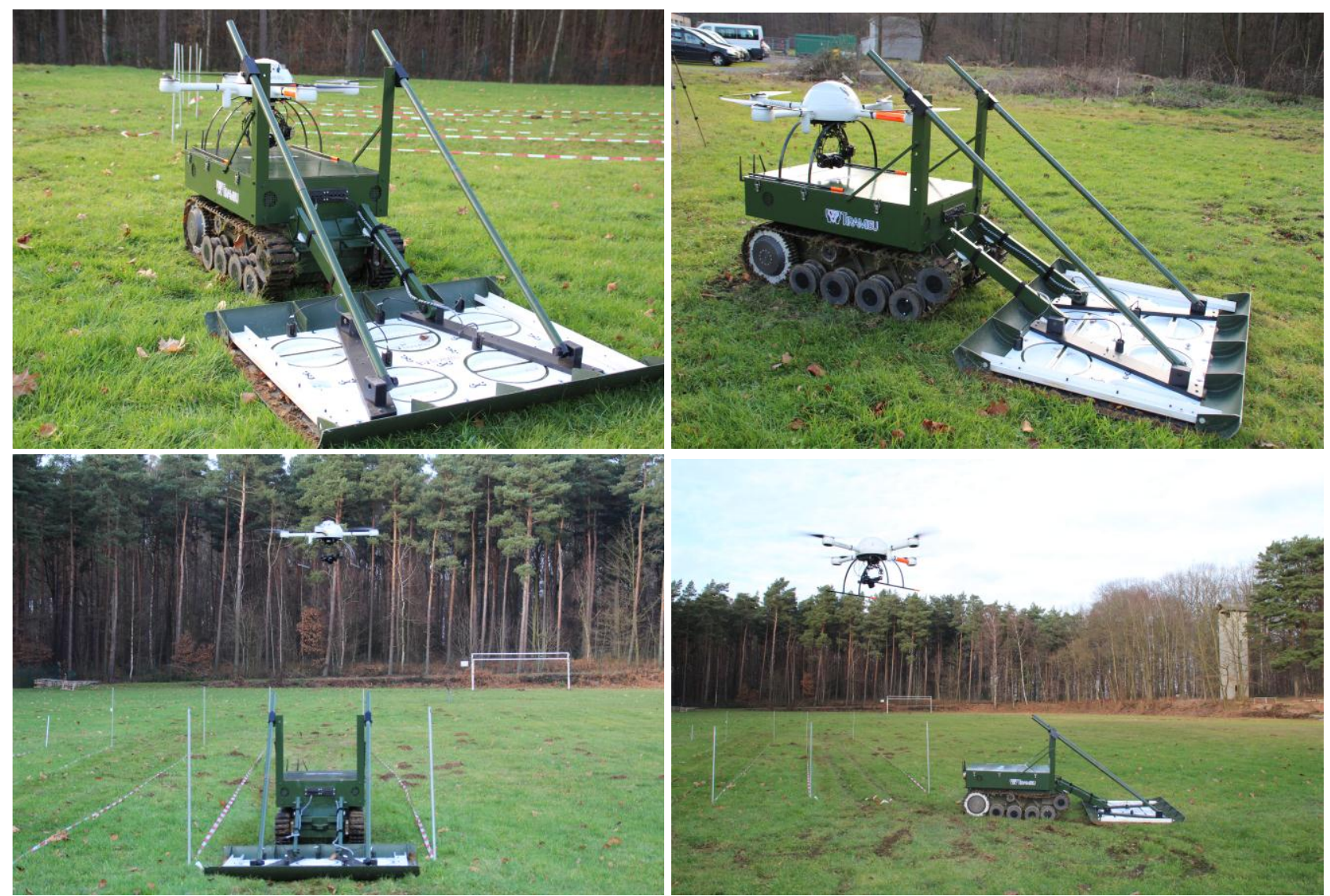

Fig. 2: Humanitarian Demining Unmanned System (UGV + UAV) 


\section{Search and Rescue Robot Platform}

The search and rescue robot platform described here was developed in the context of the EU-FP7ICARUS project [15]. The ICARUS project deals with the development of a set of integrated components to assist search and rescue teams in dealing with the difficult and dangerous, but lifesaving task of finding human survivors. The ICARUS tools consist of assistive unmanned air, ground and sea vehicles, equipped with victim detection sensors. The unmanned vehicles collaborate as a coordinated team, communicating via ad hoc cognitive radio networking. To ensure optimal human-robot collaboration, these tools are seamlessly integrated into the command and control equipment of the human crisis managers and a set of training and support tools is provided to them to learn to use the ICARUS system.
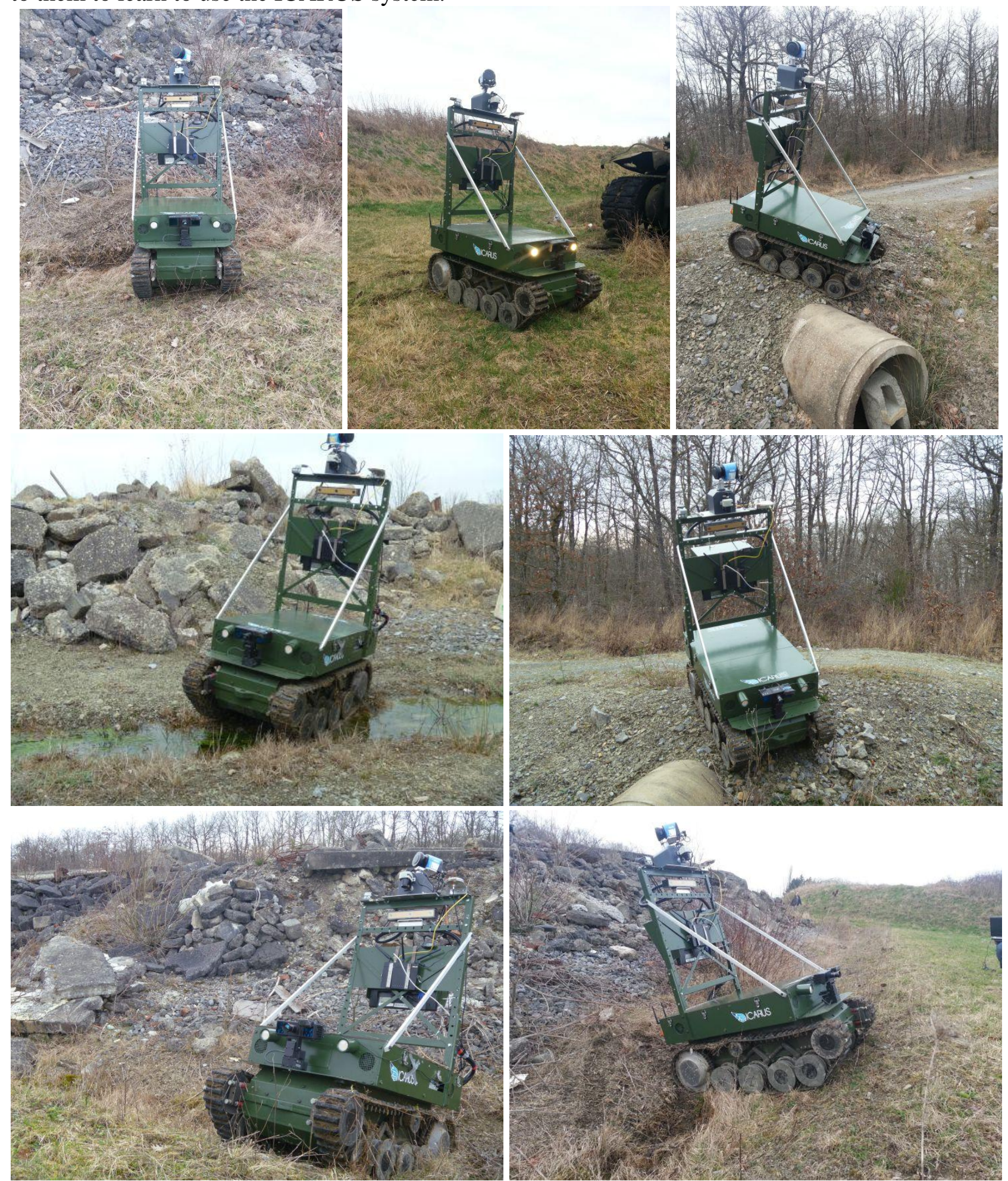
The developed search and rescue robot, as depicted in Fig. 3, is only one of the many platforms developed within the project $[15,16]$ and consists of a heavily modified Teodor chassis, equipped with an extensive sensor arsenal. This enhanced perception capability as compared to the demining application is required because in a search and rescue context it is to be expected that the robotic system will have to execute more types of missions in an autonomous or at least semi-autonomous way, where the human operator only gives high-level robot tasks or missions, which are then executed by the robotic system, relying on its autonomous perception capabilities.

As shown on Fig. 3, the following sensing modalities are used for environmental perception and navigation assistance:

- A real-time 3D laser mapping system [17]

- A real-time stereo vision system [18]

- A real-time time-of-flight camera [19]

By fusing [20] all these sensing modalities, it is possible to capture the 3D characteristics of the terrain, which is then used for autonomous navigation [6] during search and rescue missions. The system is used to explore dangerous areas and send relevant data back to the human mission planners, to increase their situational awareness.

\section{Conclusions}

In this paper, we have shown the development of 2 distinct robotic systems, starting from the same base platform. Both robotic systems - one geared towards the humanitarian demining application, the other towards search and rescue - are capable of capable of navigating in rough outdoor terrain. The demining platform is equipped with a novel metal detector array, allowing for improved landmine-detection capabilities. The search and rescue platform is equipped with a rich set of sensors, allowing the vehicle to navigate autonomously in unknown and unstructured terrain. Validation tests with end users have shown the effectiveness of both systems. The technologies developed on these validation platforms will now be ported to other platforms to enhance the application area of the developed technologies.

\section{Acknowledgements}

The research leading to these results has received funding from the European Union Seventh Framework Programme (FP7/2007-2013) under grant agreement number 285417 (ICARUS) and 284747 (TIRAMISU).

\section{References}

[1] R.R. Murphy, S. Tadokoro, D. Nardi, A. Jacoff, P. Fiorini, H. Choset, A.M. Erkmen, Search and Rescue Robotics, in Springer Handbook of Robotics, pp. 1151-1173, 2008

[2] A. Finn, A. Jacoff, M. Del Rose, B. Kania, J. Overholt, U. Silva, J. Bornstein, Evaluating autonomous ground-robots, J. Field Robotics 29(5), pp. 689-706, 2012

[3] E. Colon, G. De Cubber, H. Ping, J.C. Habumuremyi, H. Sahli, Y. Baudoin, Integrated robotic systems for Humanitarian Demining, International Journal of Advanced Robotic Systems, 4(2), 2007

[4] I. Doroftei, Y. Baudoin, A concept of walking robot for humanitarian demining, Industrial Robot: An International Journal, 39 (5), pp. 441-449, 2012 
[5] E. Colon, P. Hong, J.-C. Habumuremyi, I. Doroftei, Y. Baudoin, H. Sahli, D. Milojevic, Weemaels, An Integrated robotic system for antipersonnel mines detection, Control Engineering Practice 10, pp. 1283-1291, 2002

[6] D. Doroftei, E. Colon, G. De Cubber, A Behaviour-Based Control and Software Architecture for the Visually Guided Robudem Outdoor Mobile Robot, Journal of Automation Mobile Robotics and Intelligent Systems, 2, 2008

[7] Y. Baudoin, D. Doroftei, G. De Cubber, S.A. Berrabah, C. Pinzon, F. Warlet, J. Gancet, E. Motard, M. Ilzkovitz, L. Nalpantidis, View-finder: robotics assistance to fire-fighting services and crisis management, IEEE International Workshop on Safety, Security \& Rescue Robotics (SSRR), 2009

[8] D. Doroftei, G. De Cubber, E. Colon, Y. Baudoin, Behavior based control for an outdoor crisis management robot, Proceedings of the IARP International Workshop on Robotics for Risky Interventions and Environmental Surveillance, 2009

[9] Y. Baudoin, J. Penders, Robotics Assistance to Protection Services: Users requirements, RISE 2008, Benicàssim, Spain, January 2008

[10] D. Doroftei, A. Matos, G. De Cubber, Designing Search and Rescue robots towards realistic user requirements, Advanced Concepts in Mechanical Engineering, 2014

[11] D. Doroftei, G. De Cubber, K. Chintamani, Towards collaborative human and robotic rescue workers, 5th International Workshop on Human-Friendly Robotics (HFR2012), 2012

[12] telerob Gesellschaft für Fernhantierungstechnik mbH, EOD Robot tEODor, Product Description

[13] H. Balta, H. Wolfmayr, J. Braunstein, Y. Baudoin, Integrated Mobile Robot System for Landmine Detection, HUDEM2014

[14] Information on http://www.microdrones.com/company/media-relations/md41000_Flyer_englisch_web.pdf

[15] G. De Cubber, D. Doroftei, D. Serrano, K. Chintamani, R. Sabino, S. Ourevitch, The EUICARUS project: developing assistive robotic tools for search and rescue operations, IEEE International Symposium on Safety, Security, and Rescue Robotics (SSRR), 2013

[16] G. De Cubber, D. Serrano, K. Berns, K. Chintamani, R. Sabino, S. Ourevitch, D. Doroftei, C. Armbrust, T. Flamma, Y. Baudoin, Search and rescue robots developed by the European Icarus project, 7th Int. Workshop on Robotics for Risky Environments, 2013

[17] J. Bedkowski, A. Maslowski, G. De Cubber, Real time 3D localization and mapping for USAR robotic application, Industrial Robot: An International Journal, Vol. 39 Iss: 5, 2012

[18] M. Bertozzi, A. Broggi, E. Cardarelli, R.I. Fedriga, L. Mazzei, and P.P. Porta, VIAC Expedition Toward Autonomous Mobility, Robotics and Automation Magazine, 18(3):120-124, September 2011

[19] H. Balta, G. De Cubber, D. Doroftei, Y. Baudoin, H. Sahli, Terrain Traversability Analysis for off-road robots using Time-Of-Flight 3D Sensing, 7th IARP International Workshop on Robotics for Risky Environment - Extreme Robotics, Saint-Petersburg, Russia, October 2013

[20] G. De Cubber, D. Doroftei, Multimodal terrain analysis for an all-terrain crisis Management Robot, IARP HUDEM, 2011 